\title{
Studying the Factors of Human Carotid Atherosclerotic Plaque Rupture, by Calculating Stress/Strain in the Plaque, Based on CEUS Images: A Numerical Study
}

\author{
Zhenzhou $\mathrm{Li}^{1+}$, Yongfeng Wang ${ }^{2 t}$, Xinyin $\mathrm{Wu}^{1+}$, Xin Liu ${ }^{3}$, Shanshan Huang ${ }^{1}, \mathrm{Yi} \mathrm{He}^{4}$, \\ Shuyu Liu ${ }^{5 *}$ and Lijie Ren ${ }^{6}$ \\ ${ }^{1}$ Department of Ultrasound, Shenzhen Second People's Hospital, The First Affiliated Hospital of Shenzhen University Health \\ Science Center, Shenzhen, China, ${ }^{2}$ School of Biomedical Engineering, Sun Yat-sen University, Guangzhou, China, \\ ${ }^{3}$ Guangdong Academy Research on Virtual Reality (VR) Industry, Foshan University, Foshan, China, ${ }^{4}$ Department of \\ Neurosurgery, Shenzhen Second People's Hospital, The First Affiliated Hospital of Shenzhen University Health Science \\ Center, Shenzhen, China, ${ }^{5}$ School of Pharmacy, Sun Yat-sen University, Guangzhou, China, ${ }^{6}$ Department of Neurology, \\ Shenzhen Second People's Hospital, The First Affiliated Hospital of Shenzhen University Health Science Center, Shenzhen, \\ China
}

OPEN ACCESS

Edited by:

Jonathan Wu,

University of Windsor, Canada

Reviewed by:

Zhonghua Sun,

Curtin University, Australia

Chenchu Xu,

Western University, Canada

*Correspondence:

Shuyu Liu

Isy@mail.sysu.edu.cn

†These authors have contributed equally to this work

Received: 19 August 2020 Accepted: 11 September 2020 Published: 24 November 2020

Citation:

Li Z, Wang Y, Wu X, Liu X, Huang S, He Y, Liu S and Ren L (2020) Studying the Factors of Human Carotid Atherosclerotic Plaque Rupture, by

Calculating Stress/Strain in the

Plaque, Based on CEUS Images: A Numerical Study. Front. Neuroinform. 14:596340. doi: 10.3389/fninf.2020.596340
Carotid plaque neovascularization is one of the major factors for the classification of vulnerable plaque, but the axial force effects of the pulsatile blood flow on the plaque with neovessel and intraplaque hemorrhage was unclear. Together with the severity of stenosis, the fibrous cap thickness, large lipid core, and the neovascularization followed by intraplaque hemorrhage $(\mathrm{IPH})$ have been regarded as high-risk features of plaque rupture. In this work, the effects of these factors were evaluated on the progression and rupture of the carotid atherosclerotic plaques. Five geometries of carotid artery plaque were developed based on contrast-enhanced ultrasound (CEUS) images, which contain two types of neovessel and IPH, and geometry without neovessel and IPH. A one-way fluid-structure interaction model was applied to compute the maximum principal stress and strain in the plaque. For that hyper-elastic and non-linear material, Yeoh 3rd Order strain energy density function was used for components of the plaque. The simulation results indicated that the maximum principal stress of plaque in the carotid artery was higher when the degree of the luminal stenosis increased and the thickness of the fibrous cap decreased. The neovessels within the plaque could introduce a $2.5 \%$ increments of deformation in the plaque under the pulsatile blood flow pressure. The $\mathrm{IPH}$ also contributed to the increased risk of plaque rupture that a gain of stress was 8.983, 14.526, and $34.47 \mathrm{kPa}$ for the plaque with 50,65 , and $75 \%$, respectively, when comparing stress in the plaque with $\mathrm{IPH}$ distributed at the middle to the shoulder of the plaque. In conclusion, neovascularization in the plaque could reduce the stability of the plaque by increasing the stress within the plaque. Also, the risk of plaque rupture increased when large luminal stenosis, thin fibrous cap, and IPH were observed.

Keywords: carotid atherosclerotic plaque, vulnerable plaque, cardiovascular diseases, neovascularization, intraplaque hemorrhage, contrast-enhanced ultrasound, computational simulation 


\section{INTRODUCTION}

Carotid atherosclerotic plaque is one of the most common causes of stroke (Rossi et al., 2002; Murata et al., 2020), based on the fibrous cap, lipid core, and endothelial denudation with superficial platelet aggregation being closely associated with the incident (Sadat et al., 2009). Thin fibrous cap and large lipid core are the major factors of the vulnerable plaque and high risk of the plaque rupture (Falk et al., 1995; Naghavi et al., 2003; Finn et al., 2010), as rupture is commonly found in the plaque with the thickness of the fibrous cap $<0.065 \mathrm{~mm}$ and the volume of the lipid core accounting for $40 \%$ of the total plaque volume (Kolodgie et al., 2001). Additionally, intraplaque hemorrhage (IPH) is also considered as a risk factor for the occurrence of future cardiovascular events based on previous investigations (Takaya et al., 2006; Crombag et al., 2019; Saba et al., 2019a).

Neovascularization in the carotid atherosclerotic plaque is the maker of a high risk of the vulnerable plaque (Moreno et al., 2004; Dunmore et al., 2006; Huang et al., 2008; Van der Veken et al., 2016; Demeure et al., 2017). As early as 1999, McCarthy et al. (1999) made a histological examination of 28 patients with carotid arteries, and found that neovessels were commonly found in the fibrous cap of atherosclerotic plaque and most of them existed in the medial and lateral corners of the plaque, but rarely at the bottom. Neovessels can become leakage sites of blood vessels through the aggregation of the inflammatory cells that increase the vulnerability of the plaque, and even lead to IPH (Dunmore et al., 2006; Moreno et al., 2006). Contrast-enhanced ultrasound (CEUS) imaging has been applied to assess the neovascularization in carotid atherosclerosis plaques, for additional evaluation of the vulnerable plaque during the screening procedure for stroke in clinical practice (Feinstein, 2006; Vicenzini et al., 2007; Schinkel et al., 2020; Zamani et al., 2020). Xiong et al. (2009) reported that symptomatic patients had more intense contrast agent within the plaque than asymptomatic patients. Denmark and Marcin (2011) reported that the increased density of the neovascularization was associated with the increasing vulnerability degree of the carotid atherosclerosis plaque.

Hemodynamic blood flow plays an important role in determining plaque progression and ruptures (Radwa and Eldosoky, 2020). Computational fluid dynamics (CFD) analysis of the carotid atherosclerotic plaque has been used to assess the distribution of the superficial dynamic stress and strain of the plaque (Tang et al., 2003; Woorak et al., 2017). Li Z. et al. (2006) have performed stress analysis of the carotid plaque with various fibrous cap thickness in the geometry derived from in vivo magnetic resonance imaging (MRI) by using CFD, showing that the morphology and component of the plaque were significantly related to the stability of the plaque (Li Z. Y. et al., 2006). Impacts of the plaque eccentricity and hemodynamics environment were also related to the stress distribution in the plaque and rupture site, according to the study of Tang et al. (2003). These studies have taken into account the geometric and structural factors, including luminal stenotic degree, thin fibrous cap, large lipid core, and so on. Previous studies reported that the density of neovessels was positively associated with destabilization of the plaque based on the 2D transverse simulation (Lu et al., 2015; Guo et al., 2018, 2019). Teng et al. (2012) established a 2D transverse CFD model based on carotid artery endarterectomy slices, and four carotid plaque samples were used to analyze the local mechanical environment of the neovessels. Their results indicate that the local mechanical environment of the neovessel could lead to the occurrence of the IPH. Nevertheless, in their investigation, the effect of the carotid artery blood flow was not included, and the impact of axial force from pulsatile blood flow on the stability of the plaque was ignored.

In this study, numerical simulation with oneway fluid-structure interaction was conducted on the theoretical geometries of carotid atherosclerotic plaque with neovascularization and IPH. The stress and strain distribution in the plaque were evaluated regarding various plaque formations. The 2-dimensional axial plaque geometries were established based on the findings from the CEUS imaging. The thickness of the fibrous cap and the degrees of lumen stenosis were taken into account to study the impacts of neovessels and IPH on the destabilization of the carotid atherosclerotic plaque.

\section{METHODS}

\subsection{Geometries}

In this study, a one-way fluid-structure interaction model was performed to explore how neovessel and IPH affected carotid atherosclerotic plaque. Considering the structures of the plaque and neovessel occurring variation in different patients and even in the same one [see Figure 1: (a) is the carotid plaque with one neovessel, (b) is the one with three neovessels], 2D geometries based on CEUS images were taken into account in this study [see Figure 2: (a) is geometry with no neovessel, (b) is geometry with one neovessel, and (c) is one with three neovessels]. The structures of these geometries included carotid lumen, fibrous cap, lipid core, and different neovessels (Naghavi et al., 2003). For analysis, one inlet and outlet were necessary. The shapes of the fibrous cap and lipid core were designed by using a sinusoidal function (Li Z. Y. et al., 2006):

$$
\begin{aligned}
y_{1} & =\frac{D-D_{t}}{2} \cdot \cos (x) \\
y_{2} & =\frac{D-D_{t}}{2} \cdot(1+\cos (x))-d \\
S & =\frac{D}{D_{t}} \cdot 100 \%
\end{aligned}
$$

where $(i) \mathrm{D}$ and $D_{t}$ represent the diameter of the carotid lumen and the distance between the carotid artery wall and the top of the plaque, and (ii) S represents the degree of the carotid artery stenosis. The thickness of the fibrous cap, represented by d, plays a critical influence factor in the vulnerable plaque development (Cicha et al., 2011); different $d$ values were used $(\mathrm{d}=0.2,0.5$, and $0.0065 \mathrm{~mm}$ ), which is critical to plaque vulnerability. In this paper, we set the stenosis degree $\mathrm{S}$ at 50, 65, and 75\% (Avci et al., 2016). Moreover, the carotid artery lumen diameter of the geometry was assumed to be $10 \mathrm{~mm}$. The lengths of the carotid 

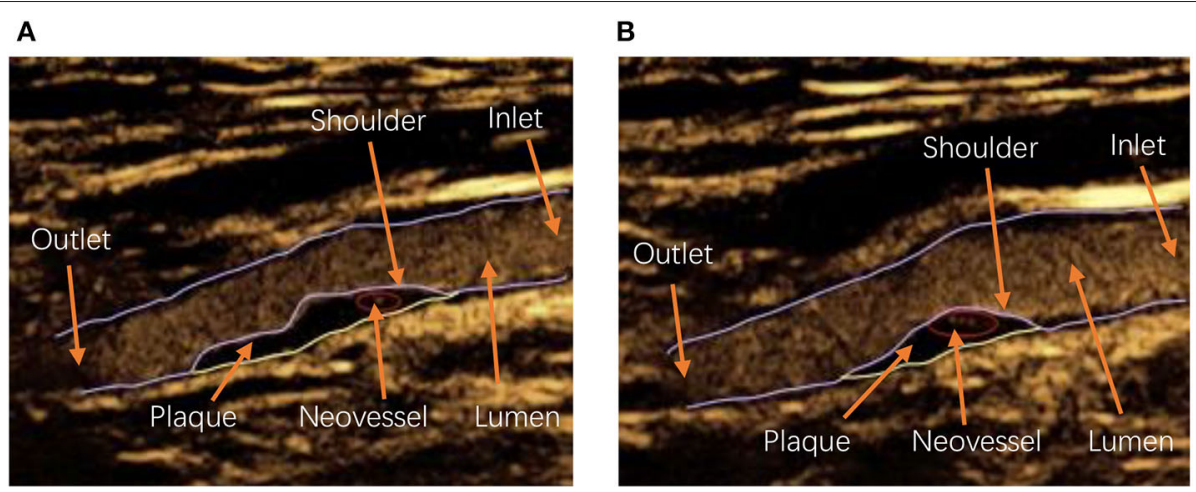

FIGURE 1 | The contrast-enhanced ultrasound (CEUS) image with carotid artery lumen, atherosclerotic plaque, and neovessel: (A) is the CEUS image with one neovessel, and (B) is the CEUS image with three neovessels. Inlet and outlet: The carotid artery blood flow inlet and outlet, respectively; Shoulder: the area at the periphery of the plaque adjacent to the normal intima beneath the fibrous cap of the plaque (Pasterkamp et al., 1998).

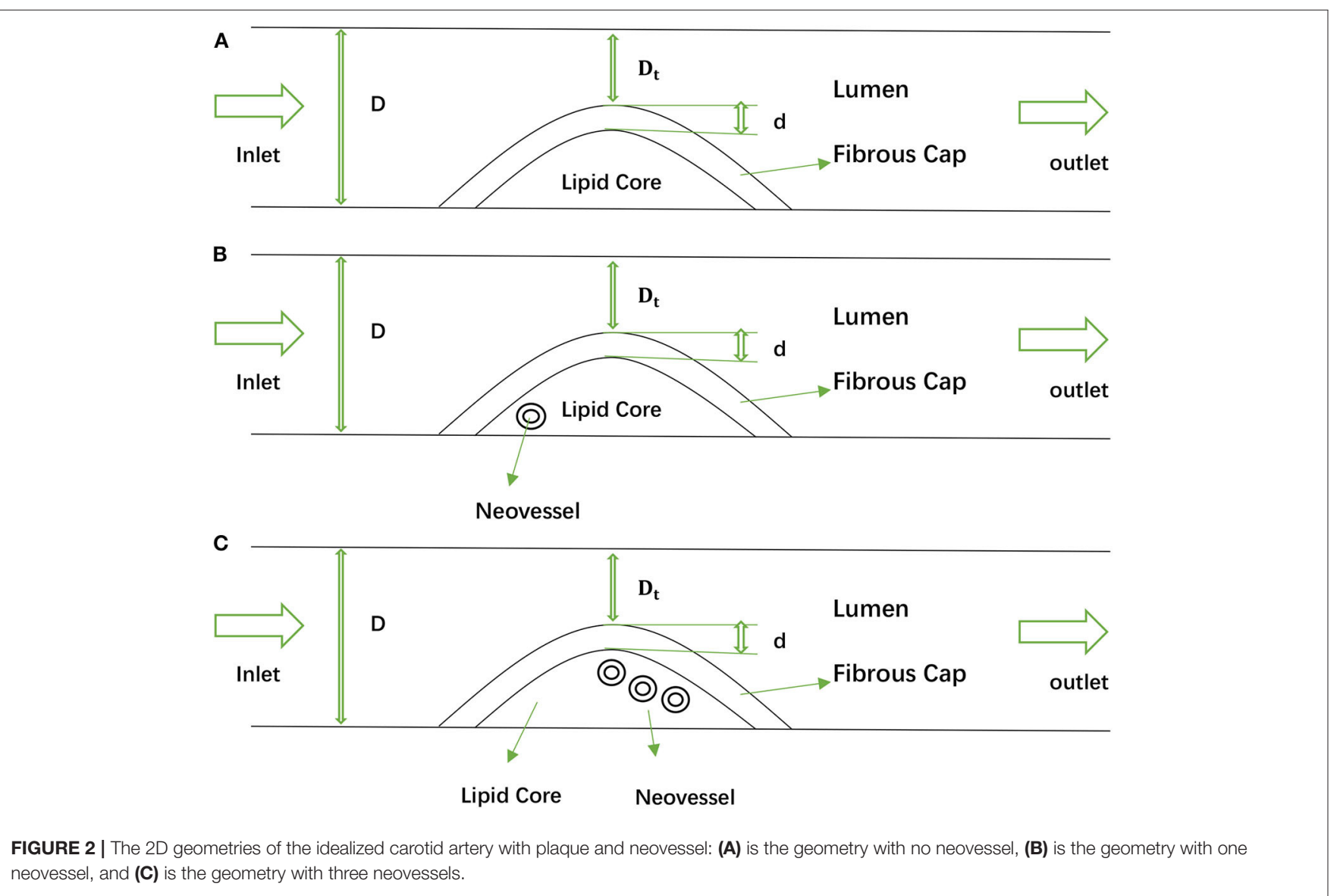

artery and the plaque are set to 100 and $20 \mathrm{~mm}$, respectively. The distance between the inlet boundary and the plaque structure was set to be $32 \mathrm{~mm}$, and the outlet boundary was set to be $48 \mathrm{~mm}$ away from the plaque structure (Belzacq et al., 2012). Additionally, the diameter of the neovessel with $0.1 \mathrm{~mm}$ thickness was set at $0.4 \mathrm{~mm}$. For the IPH, it was assumed to be a halfmoon shape (Teng et al., 2014, 2015). Two types of IPH were used in this paper with the position of one IPH set in the shoulder of plaque, and another one in the middle of the plaque (see Supplementary Figure 1).

\subsection{Computational Models, Materials, and Boundary Conditions}

In this study, the components of the plaque were assumed to be hyper-elastic and non-linear because the ideal human tissue is hyper-elastic. The Yeoh 3rd Order strain energy density function 
was used to describe the material of the plaque in the carotid artery (Teng et al., 2015).

$$
\begin{aligned}
& W=\sum_{i}^{3} C_{i}\left(I_{i}-3\right)^{i}+k(J-1), i=1,2,3 \\
& I_{1}=\lambda_{1}^{2}+\lambda_{2}^{2}+\lambda_{3}^{2}
\end{aligned}
$$

where (i) $I_{i}$ represents the first invariant of the unimodular component of the Cauchy-Green deformation tensor, (ii) $C_{i}$ is the material constants, (iii) $\lambda_{i}$ is the principal stretch, (iv) $\mathrm{J}=$ $\operatorname{def}(\mathrm{F})$ and $\mathrm{F}$ is the deformation gradient, (v) $\mathrm{k}$ is the Lagrangian multiplier for incompressibility, and (vi) $\mathrm{J}$ is equal to 0 for material incompressibility. The parameter values were chose to describe the material models (see Table 1): (i) fibrous cap: $\mathrm{C} 1=$ $53.724 \mathrm{kPa}, \mathrm{C} 2=2201.011 \mathrm{kPa}, \mathrm{C} 3=42.551 \mathrm{kpa}$, (ii) lipid core: $\mathrm{C} 1=18.548 \mathrm{kPa}, \mathrm{C} 2=207.371 \mathrm{kPa}, \mathrm{C} 3=422.652 \mathrm{kPa}$, (iii) IPH: $\mathrm{C} 1=11.225 \mathrm{kPa}, \mathrm{C} 2=69,214 \mathrm{kPa}$, and $\mathrm{C} 3=781.546 \mathrm{kPa}$, (iv) neovessel: $\mathrm{C} 1=5.656 \mathrm{kPa}, \mathrm{C} 2=1816.773 \mathrm{kPa}, \mathrm{C} 3=162.037 \mathrm{kPa}$. The density and viscosity are the fundamental blood parameters. Generally, the range of blood density is $1,030-1.070 \mathrm{~kg} / \mathrm{m}^{3}$. In our study, the constant density $1.050 \mathrm{~kg} / \mathrm{m}^{3}$ was used, and the value of the blood viscosity is $3.400 \mathrm{Ns} / \mathrm{m}^{2}$ (Marshall et al., 2004).

TABLE 1 | The parameter values of the Yeoh model used in this paper.

\begin{tabular}{lccc}
\hline & C1 (kPa) & C2 (kPa) & C3 (kPa) \\
\hline Fibrous cap & 53.724 & 2201.011 & 42.551 \\
Lipid core & 18.548 & 207.371 & 422.652 \\
Neovessel & 5.656 & 1816.773 & 162.037 \\
IPH & 11.225 & 69.214 & 781.546 \\
\hline
\end{tabular}

The blood was assumed to be an incompressible, laminar, and Newtonian fluid.

Additionally, the inlet boundary condition was transient with time (see Figure 3). This trend of the velocity changed over time, and it was close to the experimental curves (Viedma et al., 1997). After one cardiac cycle, the blood flow of the carotid outlet tended to be stable, and the relative pressure of the outlet was set as $0 \mathrm{~Pa}$. For simulating the blood flow, the conditions of no-slip and nondeformation were considered at the carotid artery wall. The 2D computational models were solved by using ANSYS2019 R1. The one-way fluid-structure interaction was operated. The pressure of the fluid was loaded in the whole plaque, and an approximate blood pressure of $0.00133 \mathrm{MPa}$ in the microvessel was exerted on the neovessel and IPH.

\section{RESULTS}

Quantification parameters were evaluated in the present study. These parameters included total deformation, maximum principal stress, and maximum principal strain. The detail of the parameters was summarized in Table 2 . The deformation of the plaques was negatively related to the thickness of the fibrous cap. The largest deformation was found in the geometry with a thin fibrous cap (thickness was $0.0065 \mathrm{~mm}$ ), and it was 0.14619 (see Figure 4C). Also, the deformation of the neovessel was in line with the observation in the CEUS images that the shape of neovessel changes along with the pulsatile blood flow, which suggested that the stress on the plaque could further induce the stress on the neovessel. Moreover, the neovessel mainly consisted of endothelial cells without smooth muscle cells, which makes the neovessel vulnerable to the stress. Additionally, the deformation of the plaque with three neovessels (50\% luminal stenosis, different thickness of fibrous cap) showed that the

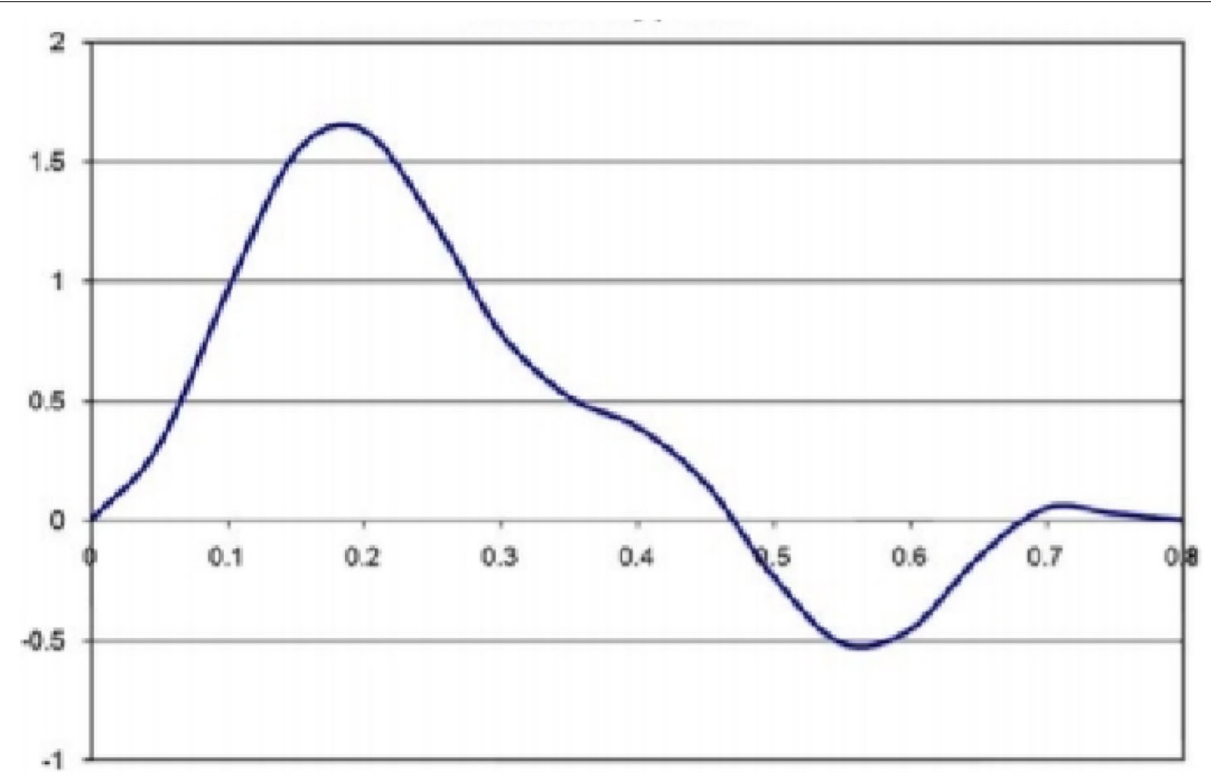

FIGURE 3 | The velocity condition specified at the inlet boundary. 
TABLE 2 | Comparison of maximum principal strain [MP strain ( $\mathrm{mm} \backslash \mathrm{mm})]$ and stress [MP stress (kPa)] of plaque during one cardiac cycle among no neovessel, one neovessel, three neovessels, and intraplaque hemorrhage (IPH).

\begin{tabular}{|c|c|c|c|c|}
\hline Degree of plaque stenosis (\%) & Thickness of fibrous cap (mm) & Plaque geometry & MP strain (mm/mm) & MP stress (kPa) \\
\hline & & No neovessel and IPH & 0.00116 & 11.6 \\
\hline & & One neovessel & 0.020946 & 11.625 \\
\hline & 2 & Three neovessels & 0.019249 & 11.565 \\
\hline & & $\mathrm{IPH}$ in plaque shoulder & 0.016795 & 11.54 \\
\hline & & $\mathrm{IPH}$ in plaque middle & 0.017509 & 11.826 \\
\hline & & No neovessel and IPH & 0.015697 & 21.276 \\
\hline & & One neovessel & 0.021508 & 21.391 \\
\hline & 0.5 & Three neovessels & 0.018977 & 21.398 \\
\hline \multirow[t]{15}{*}{50} & & $\mathrm{IPH}$ in plaque shoulder & 0.048537 & 74.321 \\
\hline & & $\mathrm{IPH}$ in plaque middle & 0.078302 & 71.435 \\
\hline & & No neovessel and IPH & 0.058363 & 276.99 \\
\hline & & One neovessel & 0.06425 & 272.81 \\
\hline & 0.0065 & Three neovessels & 0.059618 & 269.89 \\
\hline & & $\mathrm{IPH}$ in plaque shoulder & 0.052972 & 296.63 \\
\hline & & $\mathrm{IPH}$ in plaque middle & 0.058464 & 272.28 \\
\hline & & No neovessel and IPH & 0.019593 & 36.25 \\
\hline & & One neovessel & 0.021154 & 36.389 \\
\hline & 2 & Three neovessels & 0.020147 & 36.178 \\
\hline & & $\mathrm{IPH}$ in plaque shoulder & 0.019791 & 36.673 \\
\hline & & $\mathrm{IPH}$ in plaque middle & 0.032817 & 37.965 \\
\hline & & No neovessel and IPH & 0.016165 & 54.49 \\
\hline & & One neovessel & 0.02203 & 54.871 \\
\hline & 0.5 & Three neovessels & 0.018698 & 54.393 \\
\hline \multirow[t]{15}{*}{65} & & $\mathrm{IPH}$ in plaque shoulder & 0.015754 & 56.731 \\
\hline & & $\mathrm{IPH}$ in plaque middle & 0.015987 & 54.19 \\
\hline & & No neovessel and IPH & 0.066529 & 563.67 \\
\hline & & One neovessel & 0.066929 & 575.43 \\
\hline & 0.0065 & Three neovessels & 0.066642 & 569.73 \\
\hline & & $\mathrm{IPH}$ in plaque shoulder & 0.068731 & 608.05 \\
\hline & & $\mathrm{IPH}$ in plaque middle & 0.06663 & 565.72 \\
\hline & & No neovessel and IPH & 0.054892 & 225.72 \\
\hline & & One neovessel & 0.057081 & 226.87 \\
\hline & 2 & Three neovessels & 0.056651 & 225.66 \\
\hline & & $\mathrm{IPH}$ in plaque shoulder & 0.055234 & 231.15 \\
\hline & & IPH in plaque middle & 0.053922 & 226.95 \\
\hline & & No neovessel and IPH & 0.050985 & 336.36 \\
\hline & & One neovessel & 0.051242 & 335.89 \\
\hline & 0.5 & Three neovessels & 0.051038 & 333.86 \\
\hline \multirow[t]{7}{*}{75} & & $\mathrm{IPH}$ in plaque shoulder & 0.052625 & 350.08 \\
\hline & & IPH in plaque middle & 0.051274 & 336.18 \\
\hline & & No neovessel and IPH & 0.10114 & 1242.6 \\
\hline & & One neovessel & 0.10223 & 1270.3 \\
\hline & 0.0065 & Three neovessels & 0.10125 & 1250.3 \\
\hline & & $\mathrm{IPH}$ in plaque shoulder & 0.10643 & 1358.8 \\
\hline & & $\mathrm{IPH}$ in plaque middle & 0.10206 & 1261.5 \\
\hline
\end{tabular}

diameter of the neovessels was further suppressed when the fibrous cap was thin (see Figure 5). The largest deformation occurred in the plaque with $0.0065 \mathrm{~mm}$ fibrous cap thickness and the value of deformation is 0.14988 (see Figure 5C). It can be found that there was a relatively large deformation difference between the plaque with one neovessel and with three neovessels. The reason may be that the neovessel located closed to the plaque shoulder suffered from a larger pressure 


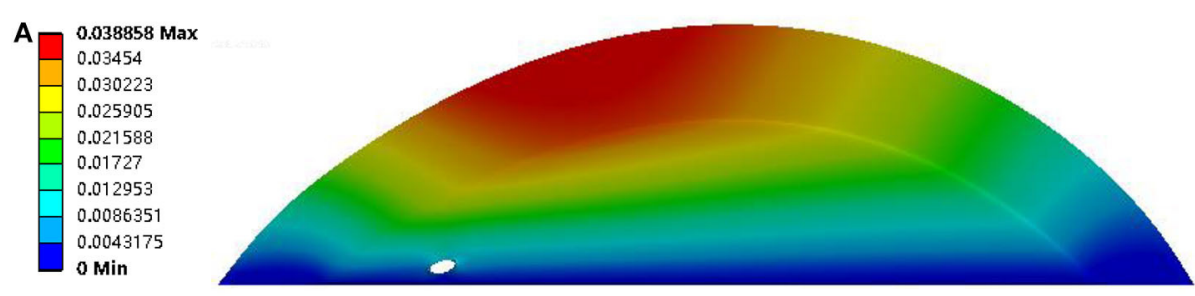

B

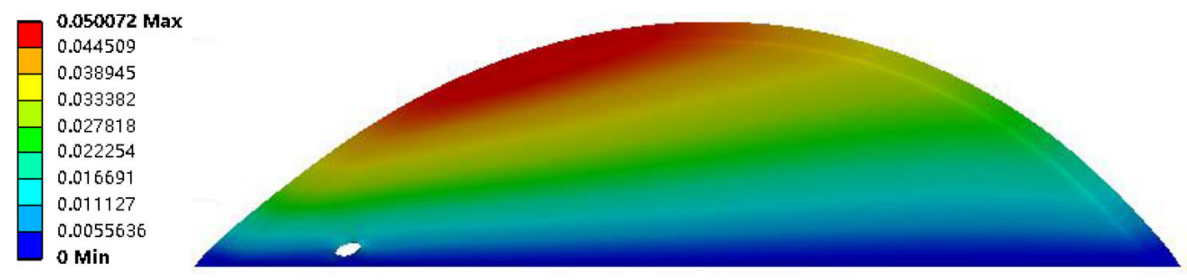

C

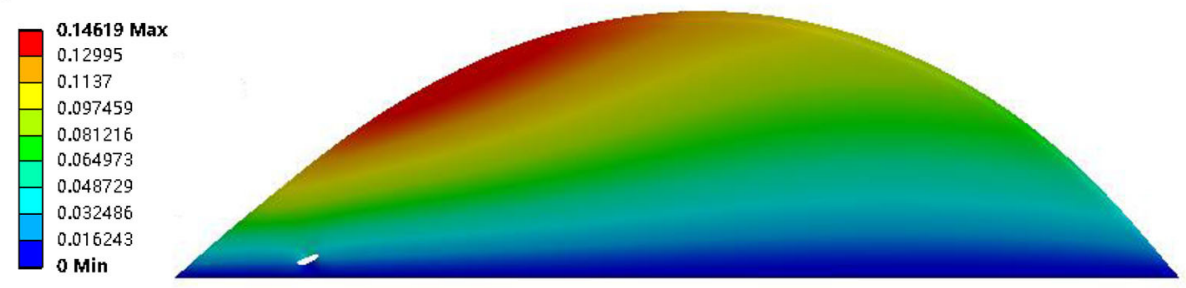

FIGURE 4 | Deformation results of plaque with 50\% luminal stenosis, one neovessel, and different thickness of the fibrous cap. The fibrous cap thicknesses of subfigures (A-C) were 2, 0.5, and $0.0065 \mathrm{~mm}$, respectively.

than the other three neovessels located in the plaque middlelower stream.

The deformation results of plaque with 65 and 75\% luminal stenoses were illustrated in the Supplementary Figures 2-5. The maximum principal stress and strain in plaque with different degrees of luminal stenosis and fibrous cap thickness are summarized in Table 2. The maximum principal stresses are recorded in the thin fibrous cap geometry that it was 276.99, 563.67 , and $1242.6 \mathrm{kPa}$ for 50,65 , and $75 \%$ luminal stenosis, respectively. A higher degree of luminal stenosis was subjected to a higher maximum principal stress and strain in all the geometries with $0.0065 \mathrm{~mm}$ fibrous cap thickness (see Figure 6). And the maximum principal stress grows along with the thinning of the fibrous cap in the geometry with $75 \%$ luminal stenosis (see Figure 7). As shown in Table 2, the largest maximum principal stress was recorded in the geometries with $75 \%$ stenosis and the thickness of fibrous cap varied from 2 to 0.0065 $\mathrm{mm}$. The maximum principal strain of plaque with $0.0065 \mathrm{~mm}$ fibrous ca thickness increased significantly with the degree of the luminal stenosis. Consequently, The plaques observed, with (i) $65 \%$ stenosis, $0.0065 \mathrm{~mm}$ thickness, and (ii) $75 \%$ stenosis, 0.5 and $0.0065 \mathrm{~mm}$ thickness, were unstable and vulnerable. Moreover, for an artery with high degree of luminal stenosis and a plaque with thin fibrous cap thickness, a higher maximum principal stress was observed when neovascularization or IPH was presented compared to those without neovascularization or IPH in most cases. And, a relatively higher maximum principal stress was observed when IPH existed in plaque shoulder than in plaque middle.

\section{DISCUSSION}

Vulnerable plaque in the carotid artery is a critical cause of many cardiovascular diseases like stroke and ischemic attacks (Schinkel et al., 2020). Neovascularization and IPH contribute significantly to the rupture of atherosclerotic plaques (Van der Veken et al., 2016; Demeure et al., 2017; Yang et al., 2020). Wang et al. (2020) found that increased carotid neovascularization was significantly related to aggravated cerebral white matter lesions in 269 participants based on CEUS. Camps-Renom et al. (2020) demonstrated that neovascularization was an independent predictor of ischemic stroke recurrence, based on 78 patients with carotid atherosclerosis. Literatures (Mark et al., 2020a,b; Yang et al., 2020) discovered that IPH had a close connection with a high risk of cardiovascular diseases. In addition, Teng et al. (2012) found that the computed parameters of stress and strain in a carotid artery plaque increased as the distance between the neovessel and the artery lumen decreased. There is a big potential for large deformation and high mechanical loading variation 


\section{A}

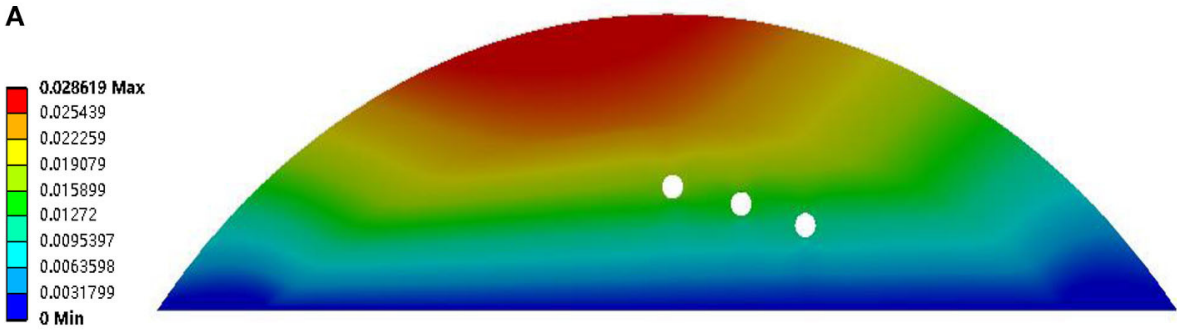

B
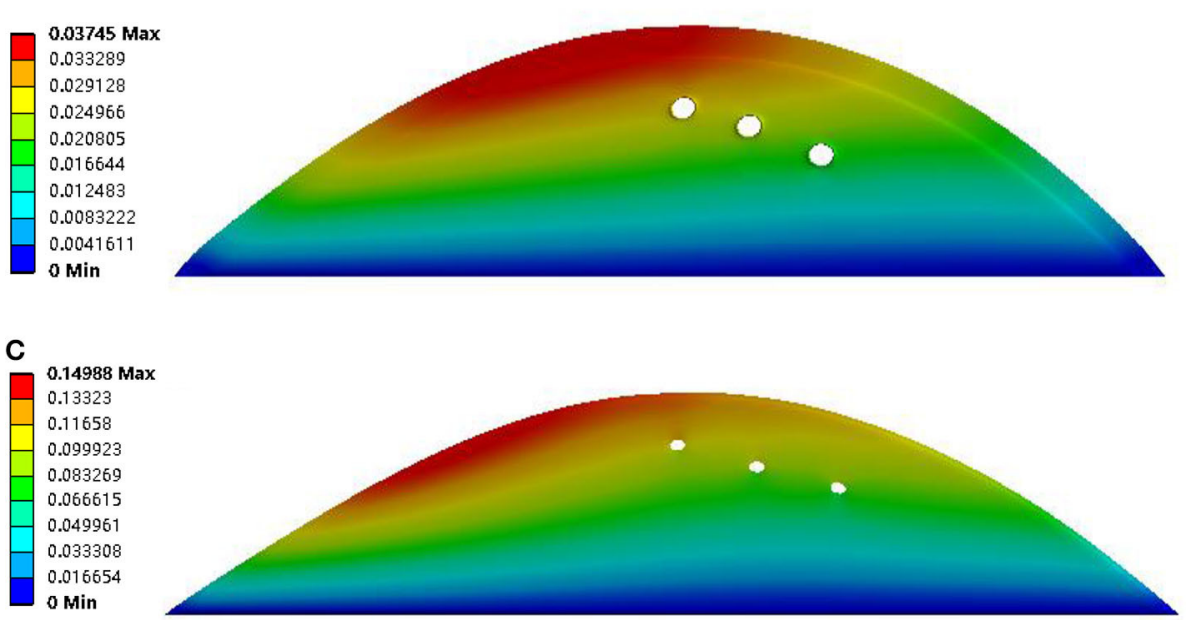

FIGURE 5 | Deformation results of plaque with 50\% luminal stenosis, three neovessel, and different thickness of fibrous cap. The fibrous cap thicknesses of subfigures (A-C) were 2, 0.5, and 0.0065, respectively.

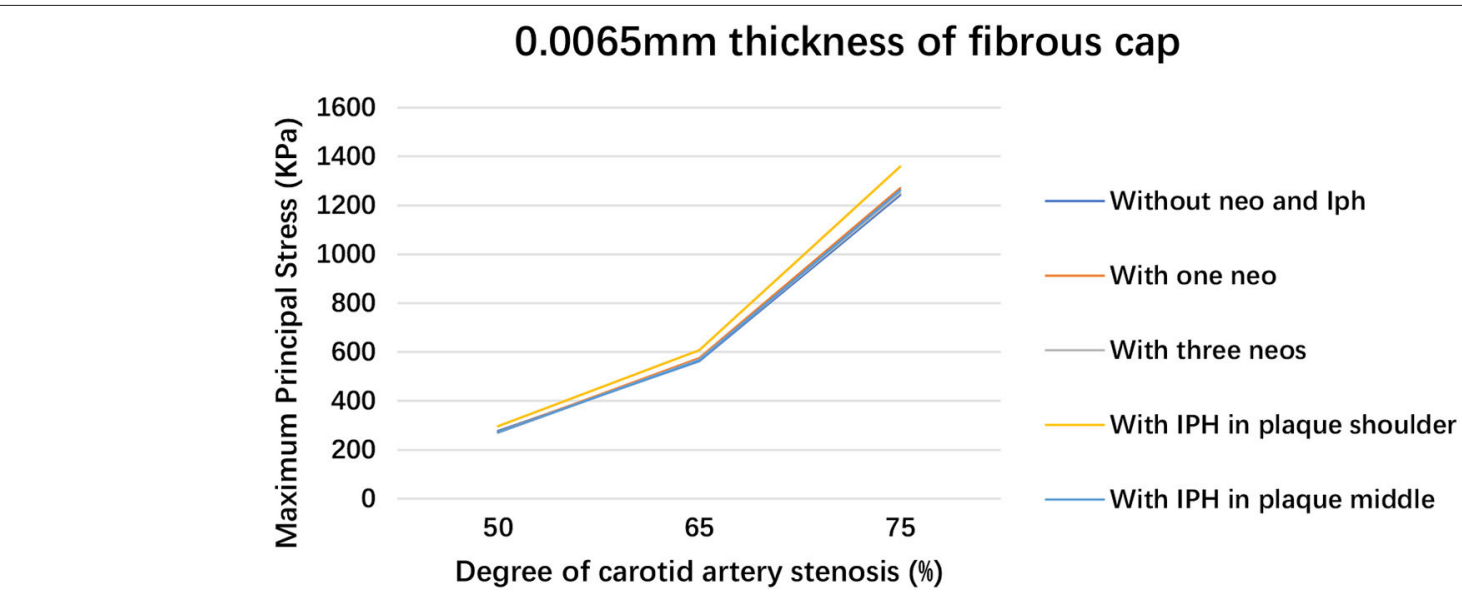

FIGURE 6 | Plaque maximum principal stress within 0.0065 mm fibrous cap thickness when changing luminal stenosis.

in an intraplaque neovessel. Huang et al. (2012) quantified the effect of IPH on plaque wall stress by using a 3D fluidstructure interaction model based on the magnetic resonance image; the authors found that the critical plaque wall stress and strain increased in geometries with IPH, relative to geometries without IPH. Additionally, fibrous cap thickness and luminal stenosis have also been demonstrated as two critical parameters that could induce plaque rupture (Li Z. Y. et al., 2006; AlegreMarténez et al., 2019; Zareh et al., 2019). A plaque with thinner fibrous cap thickness and larger lipid core was more likely to rupture and cause stroke. Huang et al. (2012) have indicated that there was a connection between fibrous cap thickness and IPH occurrence based on the analysis by fluid-structure interaction. Therefore, to be consideration of fibrous cap thickness and 


\section{$75 \%$ stenosis plaque}

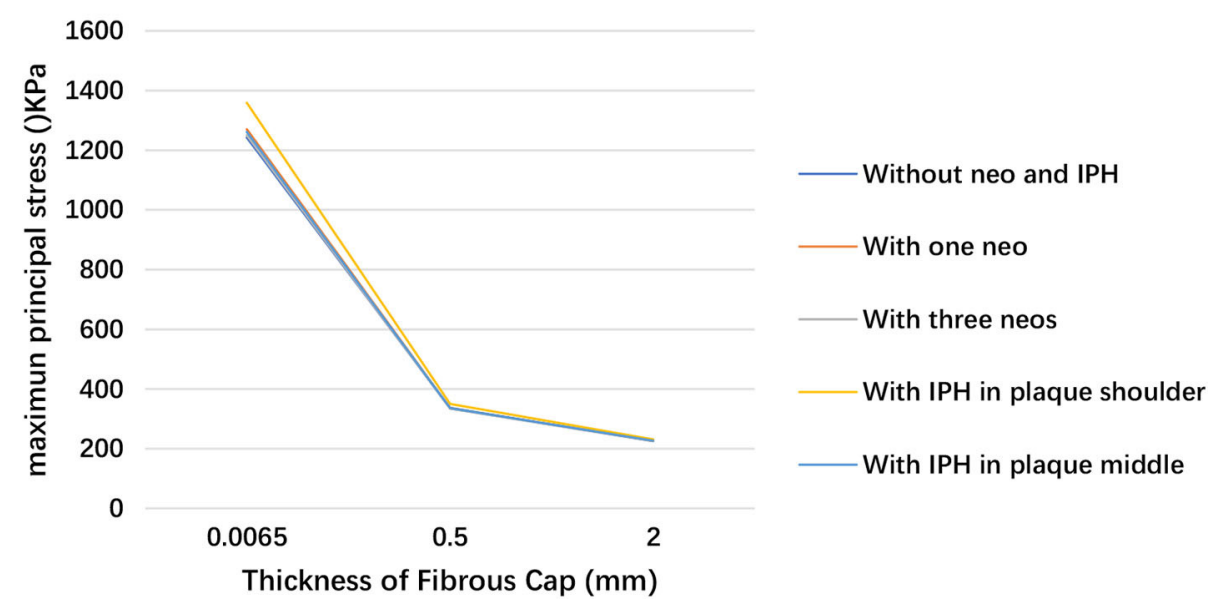

FIGURE 7 | Plaque maximum principal stress variation within 75\% stenosis luminal stenosis when changing fibrous cap thickness.

luminal stenosis are necessary for analyzing the stress and strain effect of neovascularization and IPH of plaque.

In this paper, we have constructed an idealized geometry of the carotid artery with atherosclerotic plaque based on the CEUS image. The four factors of plaque rupture, namely neovascularization, IPH, fibrous cap thickness, and luminal stenosis, were evaluated. A one-way fluid-structure interaction model was employed to calculate the structural stress and strain in the carotid atherosclerotic plaque. The simulation results validated the conclusion mentioned above that the two factors of fibrous cap thickness and luminal stenosis degree play a significant role in the progression of carotid atherosclerotic plaque. The results of the one-way fluid-structure interaction model in this paper were consistent with the current literature (Li Z. Y. et al., 2006; Teng et al., 2012). Therefore, it is reasonable to assume the role of IPH and neovascularization distributions in the formation of carotid atherosclerotic plaque. IPH is one of the important factors of carotid atherosclerotic plaque, especially in vulnerable plaque (Saba et al., 2019b). It can accelerate the enlargement of the lipid core that can increase the risk of the plaque rupture.

In our work, two types of IPHs have been evaluated, and stress and strain were determined within the plaque were observed. It was a critical indication that IPH distributed at the shoulder of the plaque showed a higher risk to rupture compared to the plaque with IPH distributed in the middle area regardless of the fibrous cap thickness and the luminal stenosis degree (see Table 2). For the plaque with IPH in the plaque shoulder, 75\% stenosis, and thin fibrous cap thicknesses, the maximum principal stress increased on an average to $34.47 \mathrm{kPa}$ compared to the plaque with IPH in the plaque middle. In the plaque with 50 and $65 \%$ stenosis, the maximum principal stress increased on an average to 8.983 and $14.526 \mathrm{kPa}$, respectively. Besides, the plaque with IPH in the plaque shoulder had a $116.3 \mathrm{kPa}$ higher maximum principal stress than the plaque without IPH, especially for the plaque with $75 \%$ stenosis and $0.0065 \mathrm{~mm}$ fibrous cap thickness. Therefore, the location of the plaque shoulder was a high-risk site for plaque rupture frequently. Additionally, IPH was not a negligible factor for assessing plaque vulnerability, and there was guidance by Bmodel ultrasonography to distinguish IPH (Gao et al., 2017; Zheng et al., 2020a,b).

Previous studies had (Huang et al., 2008; Hoogi et al., 2011; Van der Veken et al., 2016; Demeure et al., 2017) demonstrated that the feature of neovascularization also played an important role in the progression of plaque, and it may be associated with the possibility of stroke occurrence. Therefore, neovascularization within the plaque is important. The neovascularization can be visualized by CEUS imaging, and then help the cardiologist to observe the deformation of the neovascularization. However, the effect of the neovascularization on plaque risk only depended on the cardiologist's subjective diagnosis, according to the distribution and density of the IPH. Therefore, in this paper, the neovascularization was studied by modeling the neovessel geometry within the plaque. Figures 4, 5 show the deformation of the neovessel in 50\% luminal stenosis in conjunction with different fibrous cap thickness. The larger deformation of the neovessel was found with a thinner fibrous cap. The deformation of the neovessel was in line with the discovery carotid artery plaque in CEUS images. Table 2 indicates that the maximum principal stress and strain was similar between geometries with neovessel and without neovessel as a result of the similar distribution and density of the neovessels. However, Figures 4, 5 and Supplementary Figures 1-5 show the occurrence of neovessel deformation. That is to say, the neovessel underwent the loading of mechanical stress and had the potential to rupture and then lead to IPH.

Additionally, according to experimental results of fluid flow, the distribution of plaque stress and strain mainly focused on the proximal surface of the plaque, regardless of luminal 
stenosis and fibrous cap thickness. Choi et al. (2015) have found that axial plaque stress and wall shear stress in the upstream were higher than in downstream. These findings demonstrated that the site of plaque shoulder was more likely to tend to rupture because it is undergoing shear stress induced by the blood flow (Malek, 1999). The variation of shear stress could alter the characteristics of the endothelial cell, including its morphology, function, proliferation, and even vascular injury (Malek, 1999; Chatzizisis et al., 2009). Therefore, the stress and strain distribution in the proximal surface of plaque fluid flow has an important role in adjudging plaque rupture. Additionally, the neovessel was only made up of little endothelial cells and the vulnerability of neovessel was poor. Therefore, it is of significance to improve the assessment of vulnerable plaque by analyzing the effect of hemodynamic stress and strain on the plaque with neovascularization and IPH.

There are some limitations to this study. First, it was a theoretical research, and the geometry of the carotid artery was idealized. The carotid bifurcation was not taken into account, as the neovessel and the IPH were the focus of this paper. Second, only two types of neovessels were analyzed. The shape in conjunction with the location of the neovessel was flexible and variable. McCarthy et al. (1999) found that the neovessel within a plaque may exist in the medial, the lateral corners, and the bottom of the plaque. Third, the material properties of the neovessel were assumed from literatures, and this may have resulted in the parameters of maximum principal stress and strain levels being below or over the actual value. There is a small difference in the value of the maximum principal stress between the plaque with neovessel and without neovessel. The material properties, shape, and location of the neovessel may be the most likely reason for it. Another limitation is that this idealized geometry was two-dimensional. Previous studies have illustrated the histology image of the transversal slice of the plaque, indicating the distribution of microvasculature could either axial or transversal (Lu et al., 2015; Guo et al., 2018, 2019). These neovessels were with significant variation of the size of the neovessels. While previous studies focus on the axially distributed neovessel, our results suggested that the mechanical effects of transversal distributed neovessel was also important to the vulnerability of the plaque. Also, in comparison to the previous study (Teng et al., 2015), the morphology of IPH used in the present study was generally consistent with it, even if the CEUS imaging is less applicable for detecting IPH. However, as the neovessel was more complex with branches pointing toward different directions and dimensions, further study was required to establish a more realistic geometry of the plaque with 3-dimensional distribution of the microenvironment for a

\section{REFERENCES}

Alegre-Marténez, C., Choi, K. S., Tammisola, O., and McNally, D. (2019). On the axial distribution of plaque stress: influence of stenosis, severity, lipid core stiffness, lipid core length and fibrous cap stiffness. Med. Eng. Phys. 68, 76-84. doi: 10.1016/j.medengphy.2019. 02.015 more comprehensive understanding of the mechanics property of the plaque.

\section{CONCLUSION}

In conclusion, a numerical analysis of the stress and strain within carotid artery plaque was conducted. We verified that the plaque with thin fibrous cap and serious luminal stenosis has a high risk of rupture. At the same time, neovascularization and IPH were also studied. Pulsatile blood flow was related to the deformation phenomenon of neovessel occurrence in intraplaque observed in CEUS image. Also, the IPH has the potential to increase the possibility of plaque rupture. Therefore, IPH and neovascularization may also be considered to be the features for plaque progression and even rupture, besides the two key factors of fibrous cap thickness and the degree of luminal stenosis.

\section{DATA AVAILABILITY STATEMENT}

The original contributions presented in the study are included in the article/Supplementary Material, further inquiries can be directed to the corresponding author/s.

\section{AUTHOR CONTRIBUTIONS}

ZL and XW: conceptualization. YW: methodology, validation, investigation, and writing-original draft. XL: supervision, project administration, and writing - review \& editing. SL, ZL, XL, and LR: funding acquisition. SH, YH, and LR: data curation.

\section{FUNDING}

This study received financial support from Shenzhen Science and Technology Project (JCYJ20170817171836611, JCYJ20170306092258717), Natural Science Foundation of Guangdong Province, China (2019A1515011463), Scientific Research Items of Shenzhen Science and Technology Innovation Committee (No. KJYY20180703165202011), and the Major Project of Guangzhou Science and Technology of Collaborative Innovation and Industry: intelligent video detection and recognition technology in cloud computing environment (201605122151511).

\section{SUPPLEMENTARY MATERIAL}

The Supplementary Material for this article can be found online at: https://www.frontiersin.org/articles/10.3389/fninf. 2020.596340/full\#supplementary-material 
Camps-Renom, P., Prats-Sánchez, L., Casoni, F., González-de-Echávarri, J. M., Marrero-González, P., Castrillón, I., et al. (2020). Plaque neovascularization detected with contrast-enhanced ultrasound predicts ischaemic stroke recurrence in patients with carotid atherosclerosis. Eur. J. Neurol. 27, 809-816. doi: 10.1111/ene. 14157

Chatzizisis, Y. S., Coskun, A. U., Jonas, M., Edelman, E. R., Feldman, C. L., and Stone, P. H. (2009). Role of endothelial shear stress in the natural history of coronary atherosclerosis and vascular remodeling. J. Am. Coll. Cardiol. 49, 2379-2393. doi: 10.1016/j.jacc.2007.02.059

Choi, G., Lee, J. M., Kim, H. J., Park, J. B., Sankaran, S., Otake, H., et al. (2015). Coronary artery axial plaque stress and its relationship with lesion geometry: application of computational fluid dynamics to coronary CT angiography. JACC Cardiovasc. Imaging 8, 1156-1166. doi: 10.1016/j.jcmg.2015.04.024

Cicha, I., Wörner, A., Urschel, K., Beronov, K., Goppelt-Struebe, M., Verhoeven, E., et al. (2011). Carotid plaque vulnerability: a positive feedback between hemodynamic and biochemical mechanisms. Stroke 42, 3502-3510. doi: 10.1161/STROKEAHA.111.627265

Crombag, G. A. J. C., Schreuder, F. H. B. M., van Hoof, R. H. M., Truijman, M. T. B., Wijnen, N. J. A., Vöö, S. A., et al. (2019). Microvasculature and intraplaque hemorrhage in atherosclerotic carotid lesions: a cardiovascular magnetic resonance imaging study. J. Cardiovasc. Magn. Reson. 21:15. doi: 10.1186/s12968-019-0524-9

Demeure, F., Bouzin, C., Roelants, V., Bol, A., Verhelst, R., Astarci, P., et al. (2017). Head-to-head comparison of inflammation and neovascularization in human carotid plaques implications for the imaging of vulnerable plaques. Circulation 10:e005846. doi: 10.1161/CIRCIMAGING.116.005846

Denmark, S. E., and Marcin, L. R. (2011). Correlation of carotid artery atherosclerotic lesion echogenicity and severity at standard us with intraplaque neovascularization detected at contrast-enhanced us. Int. J. Med. Radiol. 258, 618-626. doi: 10.1148/radiol.10101008

Dunmore, B. J., McCarthy, M. J., Naylor, A. R., and Brindle, N. P. (2006). Carotid plaque instability and ischemic symptoms are linked to immaturity of microvessels within plaques. J. Vasc. Surg. 45, 155-159. doi: 10.1016/j.jvs.2006.08.072

Falk, E., Shah, P. K., and Fuster, V. (1995). Coronary plaque disruption. Circulation 92, 657-671. doi: 10.1161/01.CIR.92.3.657

Feinstein, S. B. (2006). Contrast ultrasound imaging of the carotid artery vasa vasorum and atherosclerotic plaque neovascularization. J. Am. Coll. Cardiol. 48, 236-243. doi: 10.1016/j.jacc.2006.02.068

Finn, A. V., Nakano, M., Narula, J., Kolodgie, F. D., and Virmani, R. (2010). Concept of vulnerable/unstable plaque. Arterioscler. Thromb. Vasc. Biol. 30, 1282-1292. doi: 10.1161/ATVBAHA.108.179739

Gao, Z., Li, Y., Sun, Y., Yang, J., Xiong, H., Zhang, H., et al. (2017). Motion tracking of the carotid artery wall from ultrasound image sequences: a nonlinear state-space approach. IEEE Trans. Med. Imaging 37, 273-283. doi: 10.1109/TMI.2017.2746879

Guo, M., Cai, Y., Yao, X., and Li, Z. (2018). Mathematical modeling of atherosclerotic plaque destabilization: role of neovascularization and intraplaque hemorrhage. J. Theor. Biol. 450, 53-65. doi: 10.1016/j.jtbi.2018.04.031

Guo, M., Yan, C., He, C., and Li, Z. (2019). Coupled modeling of lipid deposition, inflammatory response and intraplaque angiogenesis in atherosclerotic plaque. Biomed. Eng. Soc. 47, 439-452. doi: 10.1007/s10439-018-02173-1

Hoogi, A., Adam, D., Hoffman, A., Kerner, H., Reisner, S., and Gaitini, D. (2011). Carotid plaque vulnerability: quantification of neovascularization on contrastenhanced ultrasound with histopathologic correlation. Am. J. Roentgenol. 123, 1125-1132. doi: 10.2214/AJR.10.4522

Huang, P., Huang, F., Zou, C., Sun, H., Tian, X., Yang, Y., et al. (2008). Contrast-enhanced sonographic characteristics of neovascularization in carotid atherosclerotic plaques. J. Clin. Ultrasound 36, 346-351. doi: 10.1002/jcu.20448

Huang, X., Yang, C., Canton, G., Ferguson, M., Yuan, C., and Tang, D. (2012). Quantifying effect of intraplaque hemorrhage on critical plaque wall stress in human atherosclerotic plaques using three-dimensional fluid-structure interaction models. J. Biomech. Eng. 134:121004. doi: 10.1115/1.4007954

Kolodgie, F. D., Burke, A. P., Farb, A., Gold, H. K., Yuan, J., Narula, J., et al. (2001). The thin-cap fibroatheroma: a type of vulnerable plaque: the major precursor lesion to acute coronary syndromes. Curr. Opin. Cardiol. 16, 285292. doi: 10.1097/00001573-200109000-00006
Li, Z., Howarth, S., Trivedi, R. A., U-King-Im, J. M., Graves, M. J., Brown, A., et al. (2006). Stress analysis of carotid plaque rupture based on in vivo high resolution MRI. J. Biomech. 39, 2611-22. doi: 10.1016/j.jbiomech.2005.08.022

Li, Z. Y., Howarth, S. P., Tang, T., and Gillard, J. H. (2006). How critical is fibrous cap thickness to carotid plaque stability? A flow-plaque interaction model. Stroke 37, 1195-1199. doi: 10.1161/01.STR.0000217331.61083.3b

Lu, J., Duan, W., and Qiao, A. (2015). Finite element analysis of mechanics of neovessels with intraplaque hemorrhage in carotid atherosclerosis. Biomed. Eng. Online 14, S1-S3. doi: 10.1186/1475-925X-14-S1-S3

Malek, A. (1999). Hemodynamic shear stress and its role in atherosclerosis. JAMA 282:2035. doi: 10.1001/jama.282.21.2035

Mark, I. T., Nasr, D. M., Huston, J., de Maria, L., de Sanctis, P., Lehman, V. T., et al. (2020a). Embolic stroke of undetermined source and carotid intraplaque hemorrhage on MRI: a systemic review and meta-analysis. Clin. Neuroradiol. doi: 10.1007/s00062-020-00921-2. [Epub ahead of print].

Marshall, I., Zhao, S., Papathanasopoulou, P., Hoskins, P., and Xu, Y. (2004). MRI and CFD studies of pulsatile flow in healthy and stenosed carotid bifurcation models. J. Biomech. 37, 679-687. doi: 10.1016/j.jbiomech.2003.09.032

McCarthy, M. J., Loftus, I. M., Thompson, M. M., Jones, L., London, N. J., Bell, P. R., et al. (1999). Angiogenesis and the atherosclerotic carotid plaque: an association between symptomatology and plaque morphology. J. Vasc. Surg. 30, 261-268. doi: 10.1016/S0741-5214(99)70136-9

Misaki, K., Uno, T., Nambu, I., Kimura, R., Yoshikawa, A., Kamide, T., et al. (2020b). Asymptomatic carotid intraplaque hemorrhage is associated with a high risk of cerebral infarction and death after cardiovascular surgery. J. Neurol. Sci. 412:116801. doi: 10.1016/j.jns.2020.116801

Moreno, P. R., Purushothaman, K. R., Fuster, V., Echeverri, D., Truszczynska, H., Sharma, S. K., et al. (2004). Plaque neovascularization is increased in ruptured atherosclerotic lesions of human aorta: implications for plaque vulnerability. Circulation 110, 2032-2038. doi: 10.1161/01.CIR.0000143233.87854.23

Moreno, P. R., Purushothaman, K. R., Zias, E., Sanz, J., and Fuster, V. (2006). Neovascularization in human atherosclerosis. Curr. Mol. Med. 6, 457-477. doi: $10.2174 / 156652406778018635$

Murata, K., Murata, N., Chu, B., Watase, H., Hippe, D. S., Balu, N., et al. (2020). Characterization of carotid atherosclerotic plaques using 3-dimensional merge magnetic resonance imaging and correlation with stroke risk factors. Stroke 51, 475-480. doi: 10.1161/STROKEAHA.119.027779

Naghavi, M., Libby, P., Falk, E., Casscells, S. W., Litovsky, S., Rumberger, J., et al. (2003). From vulnerable plaque to vulnerable patient: a call for new definitions and risk assessment strategies: part I. Circulation 108, 1664-1672. doi: 10.1161/01.CIR.0000087480.94275.97

Pasterkamp, G., Schoneveld, A. H., van der Wal, A. C., Haudenschild, C. C., Clarijs, R. J., Becker, A. E., et al. (1998). Relation of arterial geometry to luminal narrowing and histologic markers for plaque vulnerability: the remodeling paradox. J. Am. Coll. Cardiol. 32, 655-662. doi: 10.1016/S0735-1097(98)00304-0

Radwa M. A., and Eldosoky, M. A. A. (2020). "Studying of the blood flow behavior in a stenosed carotid artery for healthy, anemic and diabetic blood," in 2020 International Conference on Innovative Trends in Communication and Computer Engineering (ITCE) (Aswan), 72-75.

Rossi, A., Franceschini, L., Fusaro, M., Cicoira, M., Eleas, A. A., Golia, G., et al. (2002). Carotid atherosclerotic plaque instability in patients with acute myocardial infarction. J. Am. Coll. Cardiol. 111, 263-266. doi: 10.1016/S0735-1097(02)81383-3

Saba, L., Micheletti, G., Brinjikji, W., Garofalo, P., Montisci, R., Balestrieri, A., et al. (2019a). Carotid intraplaque-hemorrhage volume and its association with cerebrovascular events. Am. J. Neuroradiol. 40, 1731-1737. doi: 10.3174/ajnr.A6189

Saba, L., Saam, T., Jäger, H. R., Yuan, C., Hatsukami, T. S., Saloner, D., et al. (2019b). Imaging biomarkers of vulnerable carotid plaques for stroke risk prediction and their potential clinical implications. Lancet Neurol. 18, 559-572. doi: 10.1016/S1474-4422(19) 30035-3

Sadat, U., Weerakkody, R. A., and Bowden, D. J. (2009). Hemorrhage and large lipid-rich necrotic cores are independently associated with thin or ruptures fibrous caps: an in vivo $3 \mathrm{~T}$ MRI study. Atherosclerosis 207, 434-439. doi: 10.1016/j.atherosclerosis.2009. 05.002 
Schinkel, A. F. L., Bosch, J. G., Staub, D., Adam, D., and Feinstein, S. B. (2020). Contrast-enhanced ultrasound to assess carotid intraplaque neovascularization. Ultrasound Med. Biol. 46, 466-478. doi: 10.1016/j.ultrasmedbio.2019.10.020

Takaya, N., Yuan, C., Chu, B., Saam, T., Underhill, H., Cai, J., et al. (2006). Association between carotid plaque characteristics and subsequent ischemic cerebrovascular events: a prospective assessment with MRI-initial results. J. Vasc. Surg. 44:223. doi: 10.1016/j.jvs.2006.04.013

Tang, D., Yang, C., Kobayashi, S., Zheng, J., and Vito, R. P. (2003). Effect of stenosis asymmetry on blood flow and artery compression: a threedimensional fluid-structure interaction model. Ann. Biomed. Eng. 31, 11821193. doi: 10.1114/1.1615577

Teng, Z., He, J., Degnan, A. J., Chen, S., Sadat, U., Bahaei, N. S., et al. (2012). Critical mechanical conditions around neovessels in carotid atherosclerotic plaque may promote intraplaque hemorrhage. Atherosclerosis 223, 321-326. doi: 10.1016/j.atherosclerosis.2012.06.015

Teng, Z., Yuan, J., Feng, J., Zhang, Y., Brown, A. J., Wang, S., et al. (2015). The influence of constitutive law choice used to characterize atherosclerotic tissue material properties on computing stress values in human carotid plaques. $J$. Biomech. 48, 3912-3921. doi: 10.1016/j.jbiomech.2015.09.023

Teng, Z., Zhang, Y., Huang, Y., Feng, J., Yuan, J., Lu, Q., et al. (2014). Material properties of components in human carotid atherosclerotic plaques: a uniaxial extension study. Acta Biomater. 10, 5055-5063. doi: 10.1016/j.actbio.2014.09.001

Van der Veken, B., De Meyer, G. R., and Martinet, W. (2016). Intraplaque neovascularization as a novel therapeutic target in advanced atherosclerosis. Expert Opin. Ther. Targets 20, 1247-1257. doi: 10.1080/14728222.2016. 1186650

Vicenzini, E., Giannoni, M. F., Puccinelli, F., Ricciardi, M. C., Altieri, M., Di Piero, V., et al. (2007). Detection of carotid adventitial vasa vasorum and plaque vascularization with ultrasound cadence contrast pulse sequencing technique and echo-contrast agent. Stroke 38, 2841-2843. doi: 10.1161/STROKEAHA.107.487918

Viedma, A., Jiménez-Ortiz, C., and Marco, V. (1997). Extended willis circle model to explain clinical observations in periorbital arterial flow. J. Biomech. 30, 265-272. doi: 10.1016/S0021-9290(96)00143-1

Wang, Y., Jiang, C., Huang, H., Liu, N., Wang, Y., Chen, Z., et al. (2020). Correlation of cerebral white matter lesions with carotid intraplaque neovascularization assessed by contrastenhanced ultrasound. J. Stroke Cerebrovasc. Dis. 29, 2020-104928. doi: 10.1016/j.jstrokecerebrovasdis.2020.104928

Woorak, C., Park, S. H., Huh, H. K., and Lee, S. J. (2017). Hemodynamic characteristics of flow around a deformable stenosis. J. Biomech. 61, 216-223. doi: 10.1016/j.jbiomech.2017.07.033

Xiong, L., Deng, Y. B., Zhu, Y., Liu, Y. N., and Bi, X. J. (2009). Correlation of carotid plaque neovascularization detected by using contrast-enhanced us with clinical symptoms. Radiology 251, 583-589. doi: 10.1148/radiol.2512081829

Yang, D., Liu, Y., Han, Y., Li, D., Wang, W., Li, R., et al. (2020). Signal of carotid intraplaque hemorrhage on MR T1-weighted imaging: association with acute cerebral infarct. Am. J. Neuroradiol. 41, 836-843. doi: 10.3174/ajnr.A6498

Zamani, M., Skagen, K., Scott, H., Russell, D., and Skjelland, M. (2020). Advanced ultrasound methods in assessment of carotid plaque instability: a prospective multimodal study. BMC Neurol. 20:39. doi: 10.1186/s12883-020-1620-z

Zareh, M., Katul, R., and Mohammadi, H. (2019). Mechanics of atherosclerotic plaques: effect of heart rate. Cardiovasc. Eng. Technol. 10, 344-353. doi: 10.1007/s13239-019-00413-6

Zheng, Y., Song, H., Zhang, K., Fan, J., and Liu, X. (2020b). Dynamically spatiotemporal regularized correlation tracking. IEEE Trans. Neural Netw. Learn. Syst. 31, 2336-2347. doi: 10.1109/TNNLS.2019.2929407

Zheng, Y., Sun, Y., Muhammad, K., and De Albuquerque, V. H. C. (2020a). Weighted LIC-based structure tensor with application to image content perception and processing. IEEE Trans. Ind. Inform. doi: 10.1109/TII.2020.2980577. [Epub ahead of print].

Conflict of Interest: The authors declare that the research was conducted in the absence of any commercial or financial relationships that could be construed as a potential conflict of interest.

Copyright (C) $2020 \mathrm{Li}$, Wang, Wu, Liu, Huang, He, Liu and Ren. This is an open-access article distributed under the terms of the Creative Commons Attribution License (CC $B Y)$. The use, distribution or reproduction in other forums is permitted, provided the original author(s) and the copyright owner(s) are credited and that the original publication in this journal is cited, in accordance with accepted academic practice. No use, distribution or reproduction is permitted which does not comply with these terms. 